\title{
Status and charm physics prospects at PANDA
}

\author{
Gianluigi Boca, for the PANDA ${ }^{[1]}$ Collaboration * \\ University of Pavia and INFN \\ E-mail: gianluigi.bocadpv.infn.it
}

\begin{abstract}
PANDA is an experiment that will run at the future facility FAIR, Darmstadt, Germany. A high intensity and cooled antiproton beam will collide on a fixed hydrogen or nuclear target covering center-of-mass energies between 2.2 and $5.5 \mathrm{GeV}$. PANDA addresses various physics aspects from the low energy non-perturbative region towards the perturbative regime of $\mathrm{QCD}$, for example: the formation or production of exotic non-qqbar charm meson states connected to the recently observed XYZ spectrum; the study of gluon-rich matter, such as glueballs and hybrids; the spectroscopy of the excited states of strange and charm baryons, their production cross section and their spin correlations; the behaviour of hadrons in nuclear matter; the hypernuclear physics; the electromagnetic proton form factors in the timelike region.

The PANDA experiment is designed to achieve the above mentioned physics goals with a setup with the following characteristics: an almost full solid angle acceptance; excellent tracking capabilities with high resolution (1-2 \% at $1 \mathrm{GeV} / \mathrm{c}$ in the central region); secondary vertex detection with resolution $\approx 100$ microns or better; electromagnetic calorimetry for detections of gammas and electrons up to $10 \mathrm{GeV}$; good particle identification of charge tracks (electrons, muons, pions, kaons, protons); a dedicated interchangeable central apparatus for the hypernuclear physics; detector and data acquisition system capable of working at $20 \mathrm{MHz}$ interaction rate with an intelligent software trigger that can provide maximum flexibility.
\end{abstract}

VIII International Workshop On Charm Physics

5-9 September, 2016

Bologna, Italy

${ }^{*}$ Speaker. 


\section{Introduction}

PANDA is an experiment that will run at the FAIR facility in Darmstadt (Frankfurt, Germany) in 2023. It will continue and extend the successful physic program started at Cern with LEAR and Fermilab with E760/E835. Presently the PANDA collaboration is composed by a group of more than 500 physicists from 56 institutions of 16 countries. The experiment will use a very high intensity antiproton beam with momentum ranging from $1.5 \mathrm{GeV} / c$ to $15 \mathrm{GeV} / c$, on a fixed proton target (pellet target or jet target). The range of energy in the center of mass covered goes from $\sqrt{s}=2.2$ up to $\sqrt{s}=5.5$ enabling the study a wide physics topic range. The $\bar{p}$ beam will be accumulated in the HESR storage ring in two modes : a high intensity mode, with a beam current of 8.4 $\mathrm{mA}$ and stochastic cooling leading to a luminosity of $2 \times 10^{32} \mathrm{~cm}^{-2} \mathrm{~s}^{-1}$ and $\delta p / p=10^{-4}$, and a high resolution mode with electron cooling, a current of $0.84 \mathrm{~mA}$ a luminosity of $2 \times 10^{31} \mathrm{~cm}^{-2} \mathrm{~s}^{-1}$ and $\delta p / p=10^{-5}$. The $p \bar{p}$ system can form (non-exotic) states with any $\mathrm{J}^{\mathrm{PC}}$ not just $1^{--}$as in the $e^{+} e^{-}$experiments. Consequently all the charmonium states predicted by the potential models can be studied in PANDA. The mass and width resolution of the states formed is driven essentially by the resolution of the $\bar{p}$ momentum and less by the detector performances, as demonstrated by the experience of the experiment E835 at Fermilab.

Thanks to the $2 \times 10^{32} \mathrm{~cm}^{-2} \mathrm{~s}^{-1}$ luminosity, PANDA will accumulate $8 \mathrm{pb}^{-1}$ integrated luminosity per day (assuming overall 50\% efficiency) and $10^{4} \div 10^{7} c \bar{c}$ states/day. In 6 months data taking $1.5 \mathrm{fb}^{-1}$ will be accumulated, approximately 10 times better than the Fermilab experiments E760/E835 with a better detector, with better angular coverage, with magnetic field and the ability of detecting hadronic decay modes.

For lack of space it is impossible here to describe all physics measurements feasible in PANDA and I will concentrate only on the charm physics related studies. For a more detailed description of all physics potential the interested reader can consult the PANDA Physics Book[4].

\section{The $\mathbf{X}(3872)$ and other $\mathbf{X}, \mathbf{Y}$ states lineshape scan; the $\mathbf{Z}^{ \pm}(4430)$ lineshape scan}

The charmonium states can be divided in 'conventional' $c \bar{c}$ states predicted by the quark model and 'unconventional' states whose interpretation is still far from obvious. PANDA will be able to study many of the charmonium 'unconventional' states in great detail. An example is the $\mathrm{X}(3872)$. Several hypothesis have been suggested to explain this narrow state : excited charmonium $\left(1^{3} D_{2}\right.$ or $1^{3} D_{3}$ ), a $\bar{D} D^{* 0}$ molecule, $c \bar{c} g$ hybrid. The line shape of the $\mathrm{X}(3872)$ can help distinguish between the molecule and the $q \bar{q}$ excited state models. PANDA can exploit the high resolution of the $\bar{p}$ beam to scan the $\mathrm{X}(3872)$ in formation $\left(p \bar{p} \rightarrow X(3872) \rightarrow J / \Psi \pi^{+} \pi^{-}\right)$to study the line shape(see fig. 1).

The $X(3872)$ state is not the only puzzle in the mass spectrum. PANDA can study many of the recently reported states[2] : the $Z^{ \pm}(3900), X(3940), Z^{ \pm}(4020), Z^{ \pm}(4050), X(4160), Z^{ \pm}(4250)$, $X(4260), X(4360), Z^{ \pm}(4430), X(4660)$.

The charged $\mathrm{Z}$ states have a particular importance since they are an unambiguos signature of non$q \bar{q}$ states. In PANDA they can be studied either in production, for instance in $\bar{p} p \rightarrow Z(4430)^{ \pm} \pi^{ \pm}$ with $Z(4430)^{ \pm} \rightarrow \psi(2 S) \pi^{ \pm}$or even in formation in $\bar{p} \mathrm{~d} \rightarrow Z(4430)^{ \pm} p$. In fact PANDA can use also a deuterium target (see below details on the target system) in which the neutron can be con- 

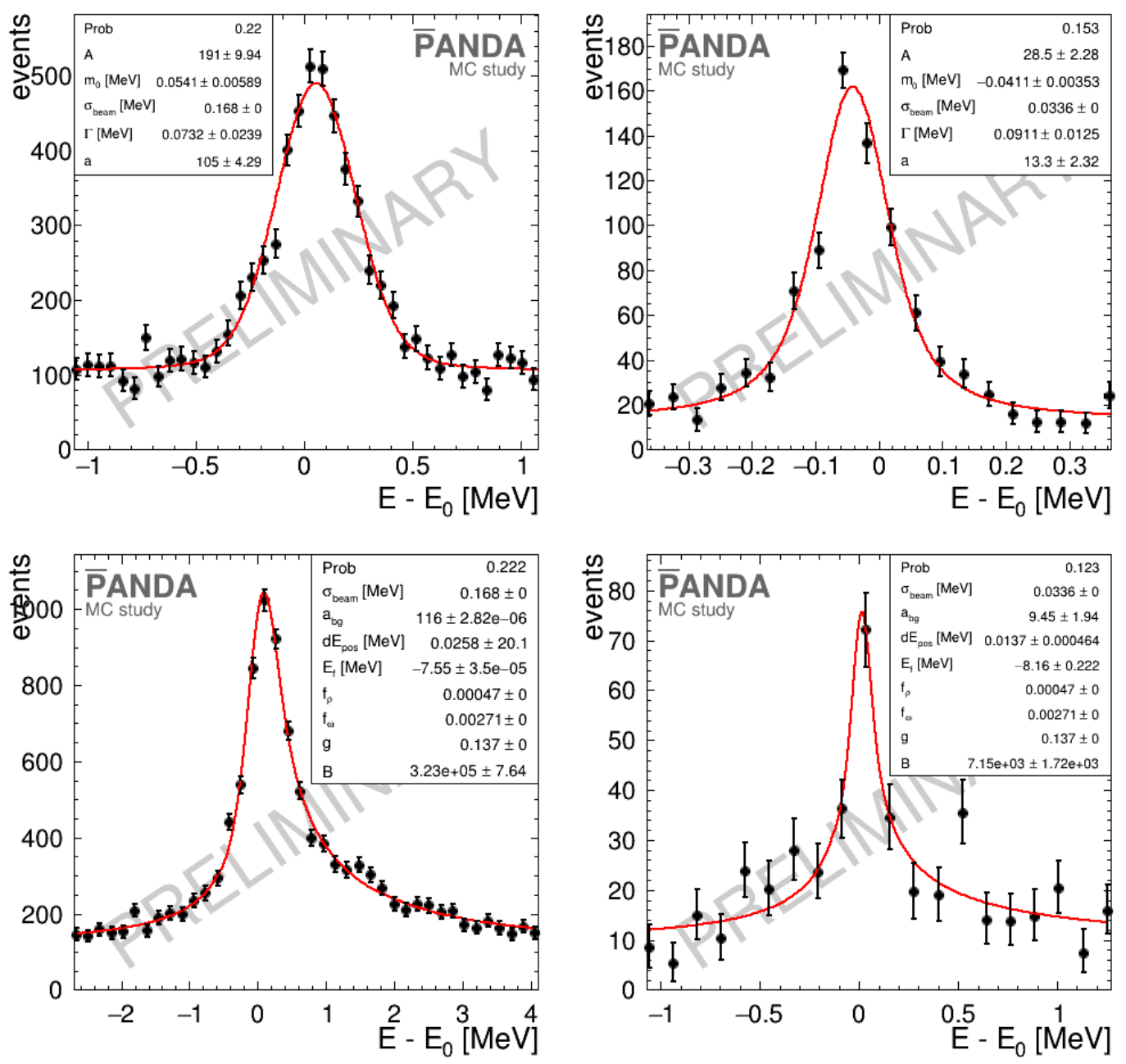

Figure 1: $\mathrm{X}(3872)$ scan examples simulated for four different scenarios. Top left plot : Breit-Wigner resonance hypothesis with $\Gamma_{x}=50 \mathrm{keV}$ in the high luminosity mode (80 days of scanning, $1.08 \mathrm{fb}^{-1}$ integrated luminosity); top right plot : Breit-Wigner resonance hypothesis with $\Gamma_{x}=100 \mathrm{keV}$ in the high resolution mode ( $46.8 \mathrm{pb}^{-1}$ integrated luminosity); bottom left : molecular model in the high luminosity mode and Flatté energy parameter $-7.5 \mathrm{MeV}$ (80 days of scanning, $1.08 \mathrm{fb}^{-1}$ integrated luminosity); bottom right plot : molecular model in the high resolution mode and Flatté energy parameter $-8.3 \mathrm{MeV}\left(46.8 \mathrm{pb}^{-1}\right.$ integrated luminosity).

sidered essentially on shell and the proton plays the role of 'spectator'. This possibility is unique to PANDA.

\section{Higher angular momentum states}

Another unique feature of PANDA is the possibility of forming or producing high angular momentum states. An example is shown in fig. 2 with the detection of an hypothetical 
$1^{3} \mathrm{~F}_{4}\left(\mathrm{~J}^{\mathrm{PC}}=4^{++}\right)$with mass $4100 \mathrm{MeV}$ state following the decay chain :

$$
\begin{aligned}
1^{3} \mathrm{~F}_{4}\left(\mathrm{~J}^{\mathrm{PC}}=4^{++}\right) & \rightarrow 1^{3} \mathrm{D}_{3}\left(\mathrm{~J}^{\mathrm{PC}}=2^{--}\right) \gamma \\
1^{3} \mathrm{D}_{3} & \rightarrow \chi_{\mathrm{c} 2}\left(\mathrm{~J}^{\mathrm{PC}}=2^{++}\right) \gamma \\
\chi_{\mathrm{c} 2} & \rightarrow \mathrm{J} / \Psi\left(\mathrm{J}^{\mathrm{PC}}=1^{--}\right) \gamma .
\end{aligned}
$$

producing a final state with a $J / \Psi$ and four $\gamma_{\text {s. }}$ Its mass can be measured with a resolution of 1.2

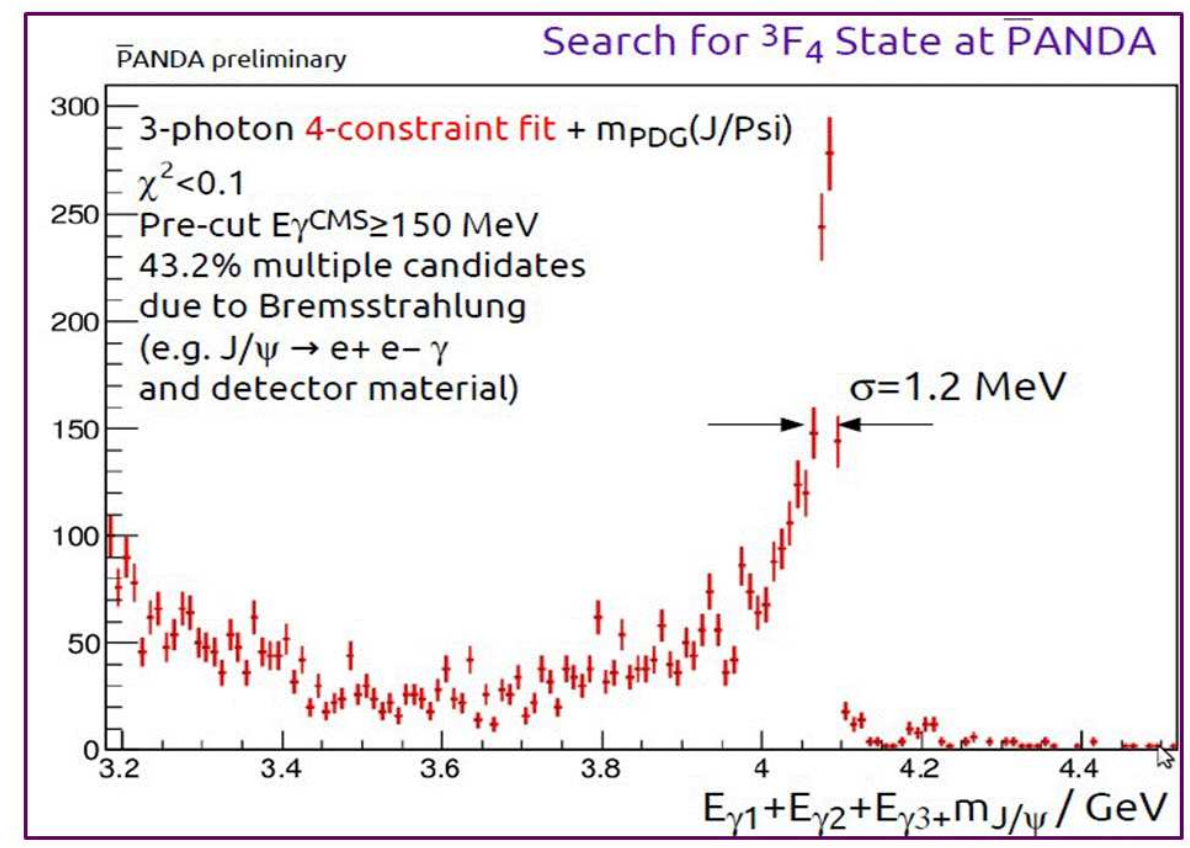

Figure 2: detection in PANDA of an hypothetical $1^{3} \mathrm{~F}_{4}(4100 \mathrm{MeV})$ state

$\mathrm{MeV}$ according to a montecarlo simulation based on a model described in [3]. That is possible thanks to the excellent $\gamma$ detection in the EM calorimeter (see below).

Another example is the detection and measurement of the $\mathrm{h}_{\mathrm{c}}^{\prime}\left(2^{1} \mathrm{P}_{1}\right)$ excited state of the $\mathrm{h}_{\mathrm{c}}$ (fig. 3) with mass $3945 \mathrm{MeV}$. This result is based on a montecarlo assuming 3 hours of data taking in PANDA.

\section{Open charm states}

Presently the large number of open charm and charm-strange states are not explained satisfactorily by the $q \bar{q}$ model. The precise measurement of their mass and width is sensitive to the different models trying to explain their properties. PANDA can measure them with unprecedented resolution by exploiting the very accurate knowledge of the momentum of the $\bar{p}$ beam. An example is the $\mathrm{D}_{\mathrm{s} 0}^{* \pm}(2317)$. At present in PDG[2] its mass is $2317.8 \pm 0.6 \mathrm{MeV}$ and its width is $<3.8 \mathrm{MeV}$. In PANDA mass and width can be measured precisely producing the $\mathrm{D}_{\mathrm{s} 0}^{* \pm}(2317)$ at threshold $: \bar{p} p \rightarrow \mathrm{D}_{\mathrm{s}}^{\mp} \mathrm{D}_{\mathrm{s} 0}^{* \pm}(2317)$. The mass of the recoil can be calculated with high resolution measuring the four-momentum of the $D_{s}^{+}$and knowing precisely the beam momentum. In fig. 4 


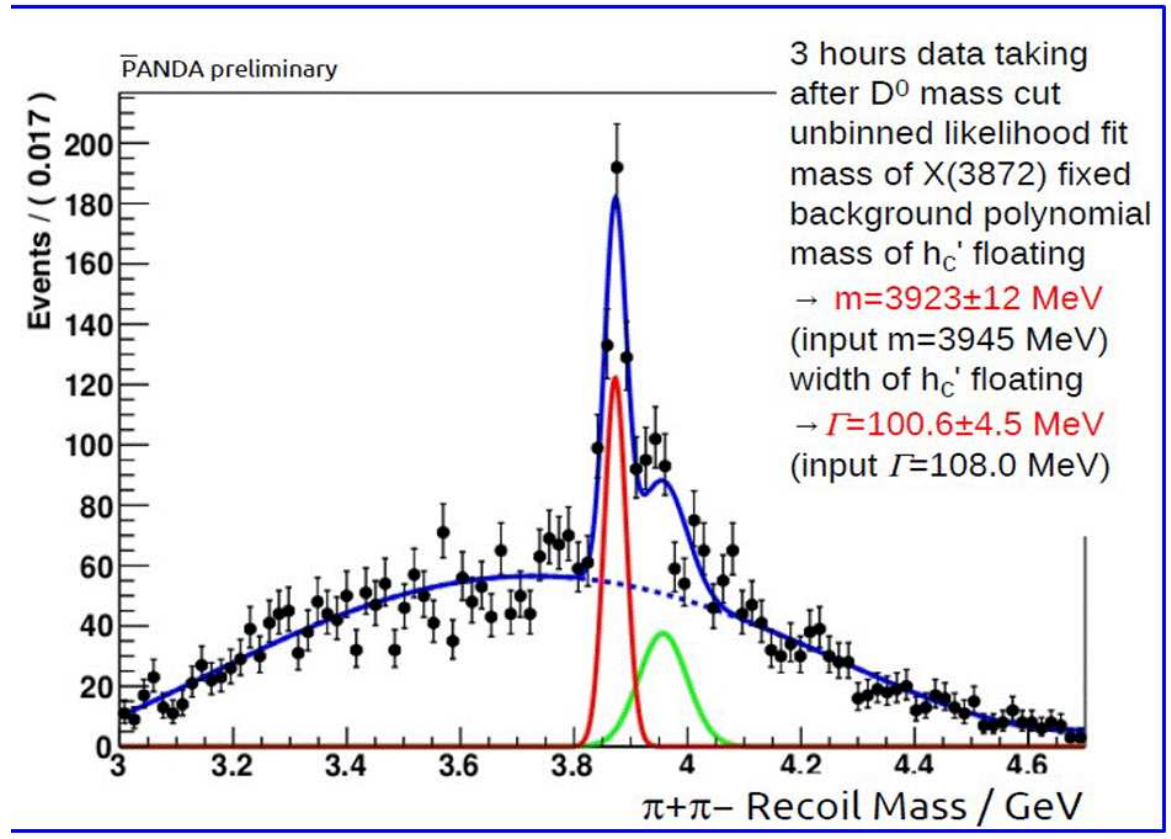

Figure 3: example of detection of $h_{c}^{\prime} 2{ }^{1} P_{1}(3945)$, a possible excited state of the $h_{c}$. The $h_{c}^{\prime}$ is the green fitted curve, the red curve is the $\mathrm{X}(3872)$ state.

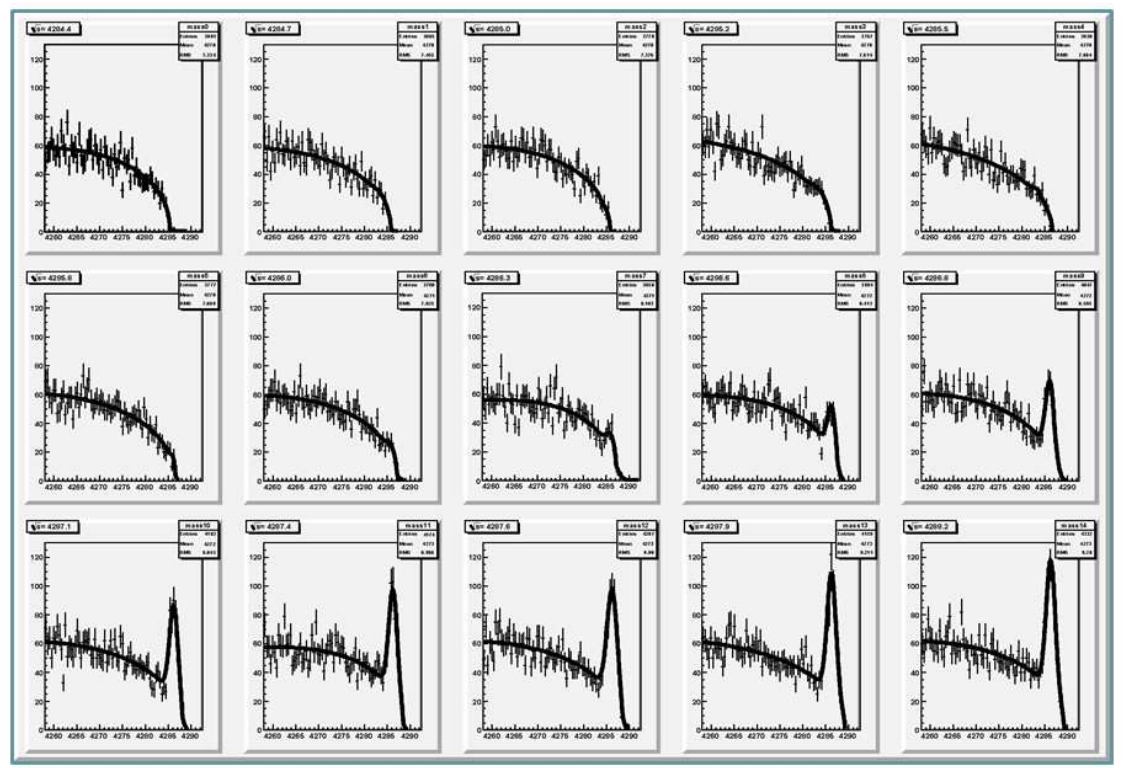

Figure 4: Montecarlo simulation of the PANDA potential of detection of $\mathrm{D}_{\mathrm{s}}(2317)$, Each plot correspond to $\sqrt{s}$ close to $4285 \mathrm{MeV}$, the production threshold of a $\mathrm{D}_{\mathrm{s}}$ plus a $\mathrm{D}_{\mathrm{s}}(2317)$. In each plot the quantity $M \equiv$ ( recoil mass to the $D_{s}^{+}$) +2317 is shown.) 
such a procedure is shown using montecarlo data that simulates the PANDA apparatus. The plots are the $\mathrm{D}_{s 0}^{* \pm}(2317)$ yields as a function of $\sqrt{s}$ of the reaction. A determination of its width better that $100 \mathrm{keV}$ can be achieved by this energy scan.

\section{The PANDA apparatus}

PANDA is a fixed target experiment and it will be performed at the accelerator facility FAIR, in Darmstad, Germany. FAIR is a complex of several accelerators (see fig 5). It will deliver different types of beam among which $\bar{p}$ accumulated in the HESR storage ring. The $\bar{p}$ will have momentum
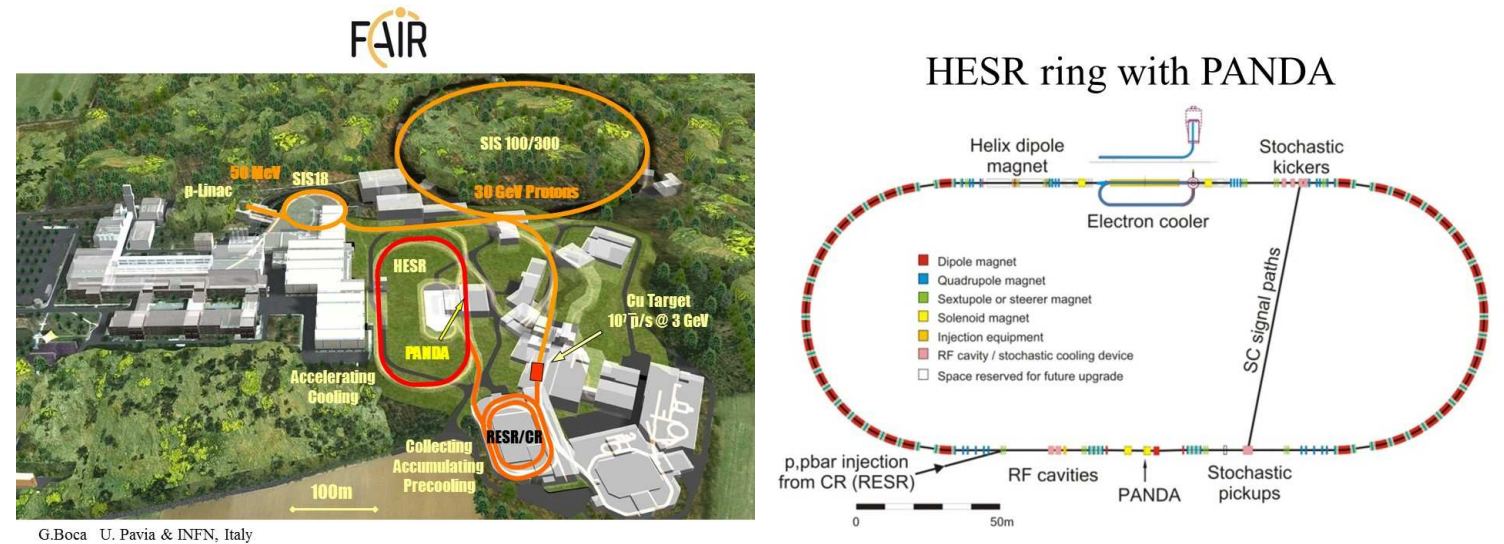

Figure 5: left plot : the FAIR accelerator complex. The experiment PANDA is on the $\bar{p}$ storage ring HESR, as shown in more detail in the right figure.

between $1.5 \mathrm{GeV} / c$ and $15 \mathrm{GeV} / c$ and will collide on the PANDA proton (or nuclear) fixed target, spanning a range from $2.2 \mathrm{GeV}$ to $5.6 \mathrm{GeV}$ of cms energy. The $\bar{p}$ beam will be cooled with an electron or stochastic cooling. In this way two running modes are possible : high intensity (HI) mode with luminosity up to a maximum $2 \times 10^{32} \mathrm{~cm}^{-2} \mathrm{sec}^{-1}$ and $\bar{p}$ momentum spread $\frac{\Delta p}{p}=10^{-4}$ and high resolution (HR) mode with luminosity a factor 10 lower and $\frac{\Delta p}{p}=10^{-5}$. In the startup phase of PANDA the interaction rate will be $2 \mathrm{MHz}$ and it will reach the maximum of $20 \mathrm{MHz}$ when FAIR accelerators are fully operative.

The PANDA apparatus is shown in fig. 6. It consists in a Central Spectrometer and a Forward Spectrometer. The Central Spectrometer has an azimuthal symmetry around the interaction point and it is embedded in a $2 \mathrm{~T}$ superconducting solenoid field. The target system blows frozen $\mathrm{H}_{2}$, deuterium or other heavier frozen gases into the $\bar{p}$ beamline. Around the interaction region the microvertex detector (MVD) is placed. Its radius goes from $2 \mathrm{~cm}$ to $16 \mathrm{~cm}$. A set of proportional straw counters (STT) sorrounds the MVD detector. Its maximum radius is $40 \mathrm{~cm}$. Going outwards a tile scintillator scintillator Time of Flight (ToF) detector is placed, then a fused silica Čerenkov detector (DIRC), the PWO crystal electromagnetic calorimeter and finally the proportional counter muon detector inside the solenoid return yoke. At the downstrean end cap region of the solenoid another DIRC disk-shaped is placed. The Central Detector covers the region with polar angle $\left(10^{\circ}<\theta<140^{\circ}\right)$. However a set of GEM detectors are placed inside the solenoid just downstream the MVD system, around the beamline, to cover polar angles from $10^{\circ}$ to 5 degrees. The region with 

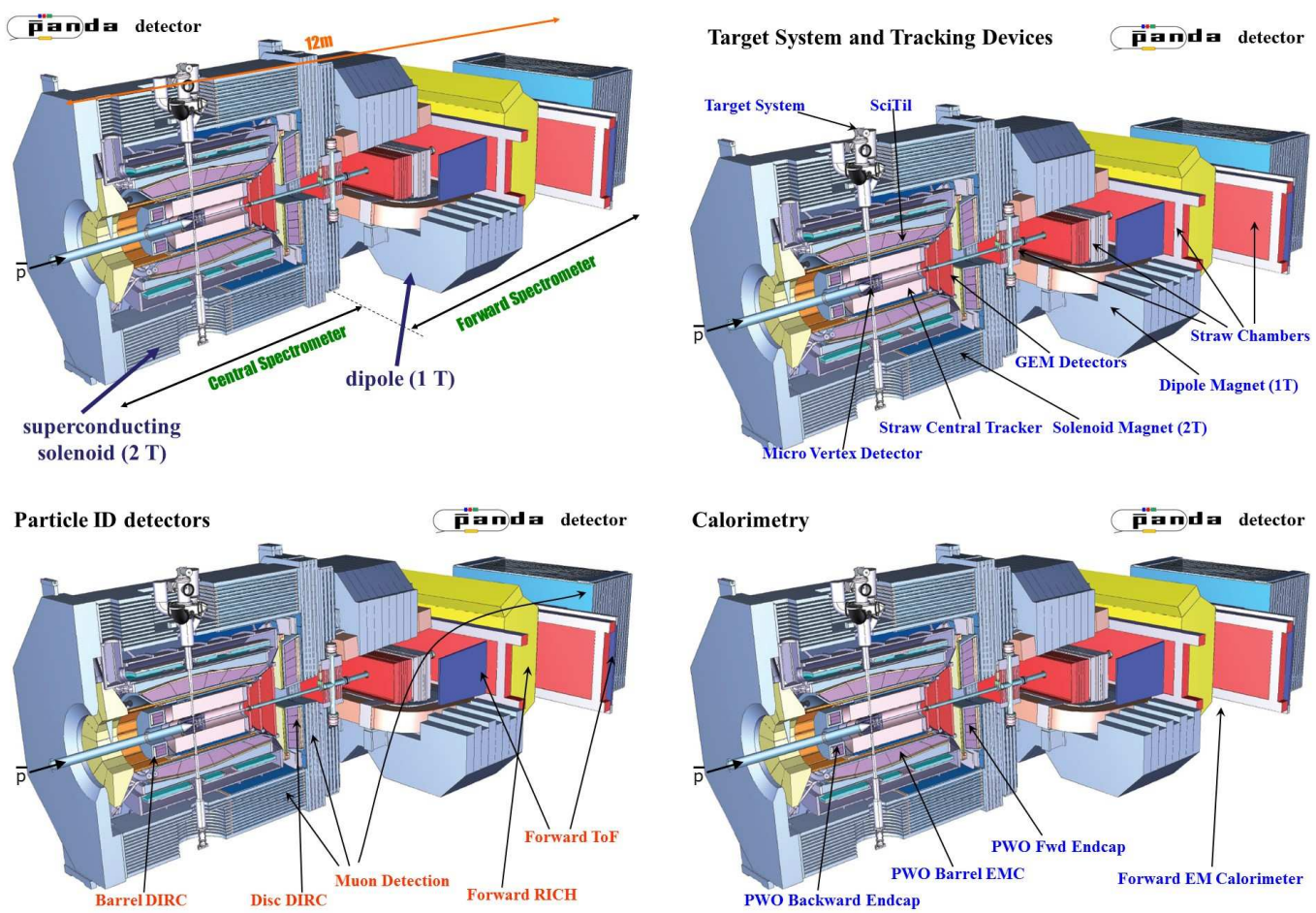

Figure 6: the PANDA detector. Top left figure : dimensions, magnet position; top right figure : the target system and the tracking devices are indicated in the Central and the Forwad Detectors; bottom left figure : the Particle IDentification detectors based on Čerenkov light detection (DIRC and RICH), on Time of Flight and the Muon detectors are indicated; bottom right figure : the ElectroMagnetic Calorimeters are indicated.

polar angle from $\approx 0$ up to $10^{\circ}$ is also covered by the Forward Spectrometer placed downstream of the Central Detector. It consist of a $2 \mathrm{Tm}$ dipole in which several stations of straw proportional chambers are placed and two scintillator walls of the forward ToF detector; just downstream the dipole another wall of ToF detectors are placed, a RICH Čerenkov, an EM calorimeter, and finally the downstream muon counters after an iron absorber. The total length of the PANDA apparatus is $12 \mathrm{~m}$.

In order to cover not only the charm physics topics described above but also a further vast range of physics not mentioned here, the PANDA spectrometer design obeys the following criteria :

- ermeticity, with an almost full solid angle coverage;

- high rate capability (from $2 \mathrm{MHz}$ at the startup up to the final $20 \mathrm{MHz}$ interaction rate);

- no hardware trigger but only a software trigger to gain maximum flexibility in the data selection. Consequently the Data Acquisition system must process and select the data at 20 $\mathrm{MHz}$;

- very good tracking with $\frac{\Delta p}{p} \sim 1-2 \%$ for $1 \mathrm{GeV} / c$ charged particles;

- vertexing capabilities for the detection of charm and strange long-lived particles; 
- excellent PID (e, $\mu, \pi, \mathrm{K}, \mathrm{p})$ achieved with Čerenkov, ToF and $\frac{d E}{d x}$ detectors;

- efficient $\gamma$ detection up to $10 \mathrm{GeV}$ energy;

- a modular design in the vertex region that will be substituted for the hypernuclear physics program. A different target and some germanium crystal detector will be used instead of the pixel and microstrip detector.

\section{Details of the experimental apparatus}

It is impossible to concentrate the description of all the PANDA detector in few pages. In this section more details are given for those detectors more relevant to the charm physics program.

\subsection{The Target System}

The fixed target consists of frozen hydrogen OR deuterium, nitrogen, neon blown into the $\bar{p}$ circulating beam at the PANDA interaction region. This technique has been used and perfectioned in the ' 90 at Fermilab and Cern. The density of the frozen target must be $5 \times 10^{15} \mathrm{~cm}^{-2}$ in order to achieve the maximum luminosity of $5 \times 10^{32} \mathrm{~cm}^{-2} \mathrm{sec}^{-1}$. The baseline design is the "pellet" target in which the frozen $\mathrm{H}_{2}$ droplets have an average diameter of $20 \mu \mathrm{m}$ and a density of $5 \times 10^{15} \mathrm{~cm}^{-2}$. A better homogeneous target can be in principle achieved by the "cluster" target design; however presently it is possible to reached the density of $1.5 \times 10^{32}$ with $2 \%$ stability. Further R\&D are underway.

\subsection{Tracking Devices in the Central Barrel}

The region around the interaction point will be covered by a MicroVertex Detector (MVD) extending from a radius of $2 \mathrm{~cm}$ up to $16 \mathrm{~cm}$ and by the proportional Straw Tube Tracker (STT) from $16 \mathrm{~cm}$ up to a radius of $40 \mathrm{~cm}$. The MVD system is made of two inner pixel barrel layers and two silicon microstrip outer barrel layers. The pixel dimension is $100 \times 100 \mu \mathrm{m}$ and their thickness is $100 \mu \mathrm{m}$ plus $200 \mu \mathrm{m}$ thickness of an ASIC preamplifier mounted on the same chip. The two outer barrels are made of double-sided microstrip chips placed at $90^{\circ}$. The system is completed by a set of 6 disks just downstream and upstream the interaction region, with pixels or wedges sensitive regions. The total number of pixel channel is 10.3 million, the total numberof strip channels is 200 thousand. The vertex tranverse resolution of the MVD system alone is estimated $50 \mu \mathrm{m}$ by a montecarlo.

The STT system consists of $\sim 5000$ straws 1.5 meter long with diameter $1 \mathrm{~cm}$, working in proportional mode. They are placed in an exagonal shape symmetric around the beam axis. All the straws are aligned with the beam axis with the exception of 8 stereo layers placed at $\pm 3^{\circ}$ for the determination of all parameters of the track helix. The straw walls are made of $27 \mu$ m mylar, filled with $\mathrm{Ar}-\mathrm{CO}_{2}$ at 1 bar overpressure, self-sustained for a total $1 \% \mathrm{X}_{0}$ material budget. A montecarlo simulation proved that the vertex resolution of this STT plus MVD system is $\sigma_{r \phi} \sim 150 \mu \mathrm{m}, \sigma_{z} \sim 1$ $\mathrm{mm}$ and a $\frac{\delta p}{p}$ resolution of $1.6 \%$ for $1 \mathrm{GeV} / c$ muon tracks.

The Central Barrel tracking system is completed by the GEM tracker that covers angles $5^{\circ}<\theta<$ $22^{\circ}$. It consists of 4 stations, each of them equipped with 6 Gas Electron Multiplier planes for 
avalanche amplification. They are filled with $\mathrm{ArCO}_{2}$. The readout is both on the cathod and on the anode, the latter shaped in circular concentric strips or radial strips for the determination of the hit position. The strip pitch goes from 200 to $300 \mu \mathrm{m}$ and the number of channels is $\sim 28000$.

\subsection{Calorimetry}

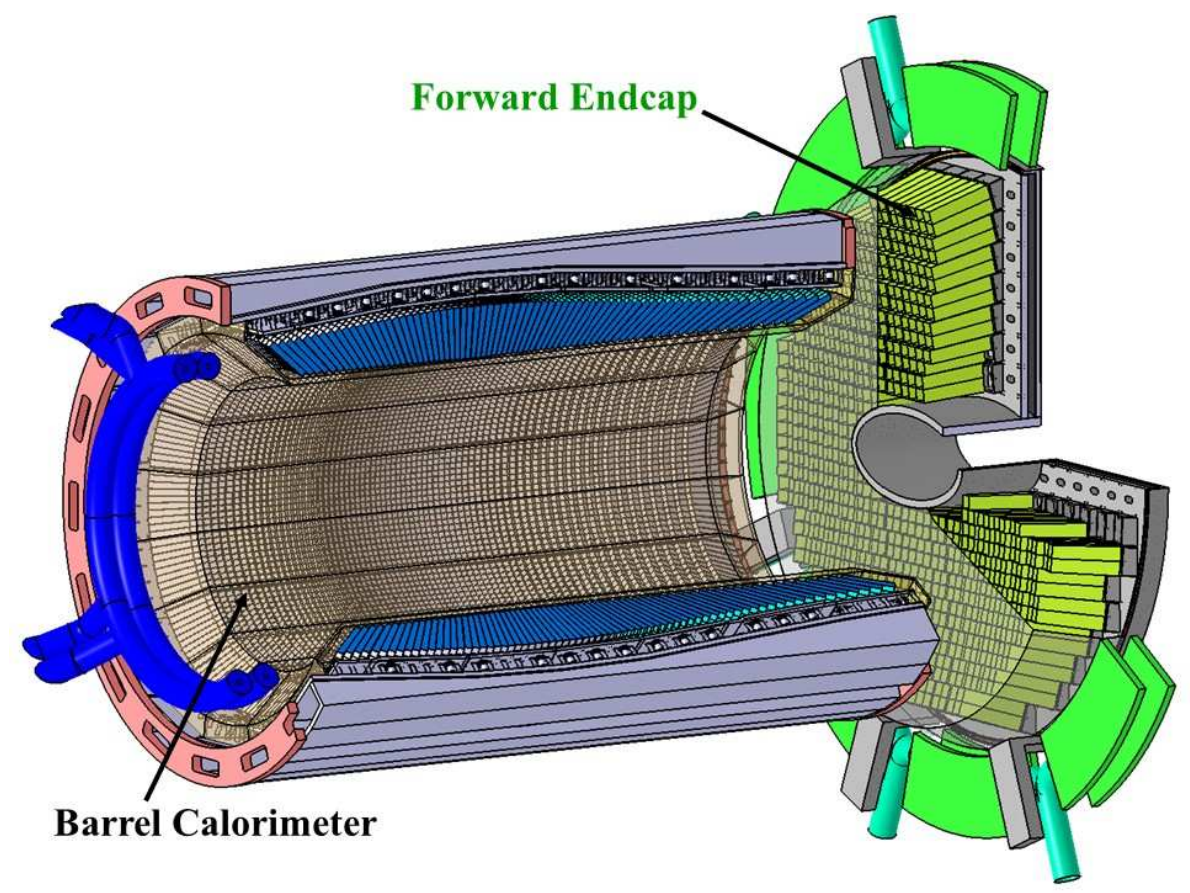

Figure 7: the Barrel Electromagnetic Calorimeter and the Forward Endcap EMC calorimeter.

The calorimetry system consist in a Central Barrel Electromagnetic calorimeter made of PWO crystals covering the entire polar angle from $\sim 10^{\circ}$ to $\sim 140^{\circ}$ degrees (fig. 7) and a Shashlyk EM calorimeter in the Forward Detector, covering the polar angle down to a couple of tenth of a degree. The Central Barrel calorimeter is divided in three sections : the Barrel ElectroMagnetic Calorimeter, the Forward Endcap EMC and the Back Endcap EMC (not shown in fig. 7). The light produced in the PWO crystals is detected by Large Area APD $2 \times 1 \mathrm{~cm}^{2}$ wide (LAAPD). The PWO crystals and the LAAPD must be kept at the temperature of $-25^{\circ}$ with a level of stability of $0.1^{\circ}$. In the Barrel EMC 11000 crystals in a projective geometry will be used, in the Forward Endcap EMC 4000. According to a montecarlo this system will ensure a resolution of $\frac{\sigma(E)}{E}=\frac{1.5 \%}{\sqrt{E}}+0.0041$ (E in $\mathrm{GeV}$ ).

\subsection{PID detectors}

Particle identification is an essential tool in PANDA to identify for instance kaons and leptons and consequently the charm particles decaying into them. Their typical momentum range in PANDA is from $200 \mathrm{MeV}$ up to $10 \mathrm{GeV}$. Several detectors are used for PID. Besides the EM calorimeters, the STT system (using the $\frac{d E}{d x}$ information), the RICH Čerenkov in the Forward detector, the Time of Flight (in the Barrel detector and in the Forward detector) the Muon Detectors and the DIRC detectors in the Barrel region. The latter are derived by the BaBar DIRC 
design and they measure the Čerenkov angle produced by charge particles traversing the fused silica bars.

\section{References}

[1] The PANDA Collaboration : B. Singh, W. Erni, B. Krusche, M. Steinacher, N. Walford, H. Liu, Z. Liu, B. Liu, X. Shen, C. Wang, J. Zhao, M. Albrecht, T. Erlen, M. Fink, F.H. Heinsius, T. Held, T. Holtmann, S. Jasper, I. Keshk, H. Koch, B. Kopf, M. Kuhlmann, M. Kümmel, S. Leiber, M. Mikirtychyants, P. Musiol, A. Mustafa, M. Pelizäus, J. Pychy, M. Richter, C. Schnier, T. Schröder, C. Sowa, M. Steinke, T. Triffterer, U. Wiedner, M. Ball, R. Beck, C. Hammann, B. Ketzer, M. Kube, P. Mahlberg, M. Rossbach, C. Schmidt, R. Schmitz, U. Thoma, M. Urban, D. Walther, C. Wendel, A. Wilson, A. Bianconi, M. Bragadireanu, M. Caprini, D. Pantea, B. Patel, W. Czyzycki, M. Domagala, G. Filo, J. Jaworowski, M. Krawczyk, E. Lisowski, F. Lisowski, M. Michałek, P. Poznański, J. Płżek, K. Korcyl, A. Kozela, P. Kulessa, P. Lebiedowicz, K. Pysz, W. Schäfer, A. Szczurek, T. Fiutowski, M. Idzik, B. Mindur, D. Przyborowski, K. Swientek, J. Biernat, B. Kamys, S. Kistryn, G. Korcyl, W. Krzemien, A. Magiera, P. Moskal, A. Pyszniak, Z. Rudy, P. Salabura, J. Smyrski, P. Strzempek, A. Wronska, I. Augustin, R. Böhm, I. Lehmann, D. Nicmorus Marinescu, L. Schmitt, V. Varentsov, M. Al-Turany, A. Belias, H. Deppe, N. Divani Veis, R. Dzhygadlo, A. Ehret, H. Flemming, A. Gerhardt, K. Götzen, A. Gromliuk, L. Gruber, R. Karabowicz, R. Kliemt, M. Krebs, U. Kurilla, D. Lehmann, S. Löchner, J. Lühning, U. Lynen, H. Orth, M. Patsyuk, K. Peters, T. Saito, G. Schepers, C.J. Schmidt, C. Schwarz, J. Schwiening, A. Täschner, M. Traxler, C. Ugur, B. Voss, P. Wieczorek, A. Wilms, M. Zühlsdorf, V. Abazov, G. Alexeev, V.A. Arefiev, V. Astakhov, M. Yu. Barabanov, B.V. Batyunya, Y. Davydov, V. Kh. Dodokhov, A. Efremov, A. Fechtchenko, A.G. Fedunov, A. Galoyan, S. Grigoryan, E.K. Koshurnikov, Y. Yu. Lobanov, V.I. Lobanov, A.F. Makarov, L.V. Malinina, V. Malyshev, A.G. Olshevskiy, E. Perevalova, A.A. Piskun, T. Pocheptsov, G. Pontecorvo, V. Rodionov, Y. Rogov, R. Salmin, A. Samartsev, M.G. Sapozhnikov, G. Shabratova, N.B. Skachkov, A.N. Skachkova, E.A. Strokovsky, M. Suleimanov, R. Teshev, V. Tokmenin, V. Uzhinsky, A. Vodopianov, S.A. Zaporozhets, N.I. Zhuravlev, A. Zinchenko, A.G. Zorin, D. Branford, D. Glazier, D. Watts, M. Böhm, A. Britting, W. Eyrich, A. Lehmann, M. Pfaffinger, F. Uhlig, S. Dobbs, K. Seth, A. Tomaradze, T. Xiao, D. Bettoni, V. Carassiti, A. Cotta Ramusino, P. Dalpiaz, A. Drago, E. Fioravanti, I. Garzia, M. Savrie, V. Akishina, I. Kisel, G. Kozlov, M. Pugach, M. Zyzak, P. Gianotti, C. Guaraldo, V. Lucherini, A. Bersani, G. Bracco, M. Macri, R.F. Parodi, K. Biguenko, K.T. Brinkmann, V. Di Pietro, S. Diehl, V. Dormenev, P. Drexler, M. Düren, E. Etzelmüller, M. Galuska, E. Gutz, C. Hahn, A. Hayrapetyan, M. Kesselkaul, W. Kühn, T. Kuske, J.S. Lange, Y. Liang, V. Metag, M. Moritz, M. Nanova, S. Nazarenko, R. Novotny, T. Quagli, S. Reiter, A. Riccardi, J. Rieke, C. Rosenbaum, M. Schmidt, R. Schnell, H. Stenzel, U. Thöring, T. Ullrich, M.N. Wagner, T. Wasem, B. Wohlfahrt, H.G. Zaunick, E. Tomasi-Gustafsson, D. Ireland, G. Rosner, B. Seitz, P.N. Deepak, A. Kulkarni, A. Apostolou, M. Babai, M. Kavatsyuk, P.J. Lemmens, M. Lindemulder, H. Loehner, J. Messchendorp, P. Schakel, H. Smit, M. Tiemens, J.C. van der Weele, R. Veenstra, S. Vejdani, K. Dutta, K. Kalita, A. Kumar, A. Roy, H. Sohlbach, M. Bai, L. Bianchi, M. Büscher, L. Cao, A. Cebulla, R. Dosdall, A. Gillitzer, F. Goldenbaum, D. Grunwald, A. Herten, Q. Hu, G. Kemmerling, H. Kleines, A. Lai, A. Lehrach, R. Nellen, H. Ohm, S. Orfanitski, D. Prasuhn, E. Prencipe, J. Pütz, J. Ritman, S. Schadmand, T. Sefzick, V. Serdyuk, G. Sterzenbach, T. Stockmanns, P. Wintz, P. Wüstner, H. Xu, A. Zambanini, S. Li, Z. Li, Z. Sun, V. Rigato, L. Isaksson, P. Achenbach, O. Corell, A. Denig, M. Distler, M. Hoek, A.

Karavdina, W. Lauth, H. Merkel, U. Müller, J. Pochodzalla, S. Sanchez, S. Schlimme, C. Sfienti, M. Thiel, H. Ahmadi, S. Ahmed, S. Bleser, L. Capozza, M. Cardinali, A. Dbeyssi, M. Deiseroth, F. Feldbauer, M. Fritsch, B. Fröhlich, D. Kang, D. Khaneft, R. Klasen, H.H. Leithoff, D. Lin, F. Maas, S. Maldaner, M. Martinez, M. Michel, M. C. Mora Espi, C. Morales Morales, C. Motzko, F. Nerling, O. 
Noll, S. Pflüger, A. Pitka, D. Rodriguez Piñeiro, A. Sanchez-Lorente, M. Steinen, R. Valente, T. Weber, M. Zambrana, I. Zimmermann, A. Fedorov, M. Korjik, O. Missevitch, A. Boukharov, O. Malyshev, I. Marishev, V. Balanutsa, P. Balanutsa, V. Chernetsky, A. Demekhin, A. Dolgolenko, P. Fedorets, A. Gerasimov, V. Goryachev, V. Chandratre, V. Datar, D. Dutta, V. Jha, H. Kumawat, A.K. Mohanty, A. Parmar, B. Roy, G. Sonika, C. Fritzsch, S. Grieser, A.K. Hergemöller, B. Hetz, N. Hüsken, A. Khoukaz, J.P. Wessels, K. Khosonthongkee, C. Kobdaj, A. Limphirat, P. Srisawad, Y. Yan, A. Yu. Barnyakov, M. Barnyakov, K. Beloborodov, V.E. Blinov, V.S. Bobrovnikov, I.A. Kuyanov, K. Martin, A.P. Onuchin, S. Serednyakov, A. Sokolov, Y. Tikhonov, A.E. Blinov, S. Kononov, E.A. Kravchenko, E. Atomssa, R. Kunne, B. Ma, D. Marchand, B. Ramstein, J. van de Wiele, Y. Wang, G. Boca, S. Costanza, P. Genova, P. Montagna, A. Rotondi, V. Abramov, N. Belikov, S. Bukreeva, A. Davidenko, A. Derevschikov, Y. Goncharenko, V. Grishin, V. Kachanov, V. Kormilitsin, A. Levin, Y. Melnik, N. Minaev, V. Mochalov, D. Morozov, L. Nogach, S. Poslavskiy, A. Ryazantsev, S. Ryzhikov, P. Semenov, I. Shein, A. Uzunian, A. Vasiliev, A. Yakutin, U. Roy, B. Yabsley, S. Belostotski, G. Gavrilov, A. Izotov, S. Manaenkov, O. Miklukho, D. Veretennikov, A. Zhdanov, T. Bäck, B. Cederwall, K. Makonyi, M. Preston, P.E. Tegner, D. Wölbing, A.K. Rai, S. Godre, D. Calvo, S. Coli, P. De Remigis, A. Filippi, G. Giraudo, S. Lusso, G. Mazza, M. Mignone, A. Rivetti, R. Wheadon, A. Amoroso, M.P. Bussa, L. Busso, F. De Mori, M. Destefanis, L. Fava, L. Ferrero, M. Greco, J. Hu, L. Lavezzi, M. Maggiora, G. Maniscalco, S. Marcello, S. Sosio, S. Spataro, F. Balestra, F. Iazzi, R. Introzzi, A. Lavagno, J. Olave, R. Birsa, F. Bradamante, A. Bressan, A. Martin, H. Calen, W. Ikegami Andersson, T. Johansson, A. Kupsc, P. Marciniewski, M. Papenbrock, J. Pettersson, K. Schönning, M. Wolke, B. Galnander, J. Diaz, V. Pothodi Chackara, A. Chlopik, G. Kesik, D. Melnychuk, B. Slowinski, A. Trzcinski, M. Wojciechowski, S. Wronka, B. Zwieglinski, P. Bühler, J. Marton, D. Steinschaden, K. Suzuki, E. Widmann, J. Zmeskal, K.M. Semenov-Tian-Shansky

[2] C.Patrignani et al. (Particle Data Group), Chinese Physics C, 40, 100001 (2016).

[3] E.S. Swanson et al, Phys.Rev.D71 (2005) 12005.

[4] M.F.M. Lutz et al.[PANDA Collaboration]; "Physics Performance Report for PANDA : Strong Interaction Studies with Antiprotons" arXiv:0903.3905; (2009). 